\title{
Variations pluviométriques durant la dernière période séculaire en Europe Occidentale
}

\author{
Changes in rainfall conditions in Western Europe \\ over the last century
}

\author{
S. Martin, J. Mounier et J. P. Marchand \\ CNRS Equipe de Recherche $n^{\circ} 30$, Grenoble
}

\section{Introduction}

Cette étude a pour but la description des variations pluviométriques survenues au cours du siècle qui vient de s'écouler, pour un ensemble de stations réparties sur la façade atlantique de l'Europe de l'Ouest et du Sud-Ouest. A partir de la documentation climatique disponible, il s'agit par une étude comparée des séries de mesures annuelles puis saisonnières de dégager les principales variations communes à un grand espace géographique.

Dans cet espace européen, la documentation concernant les stations retenues (Fig. 1) a été empruntée

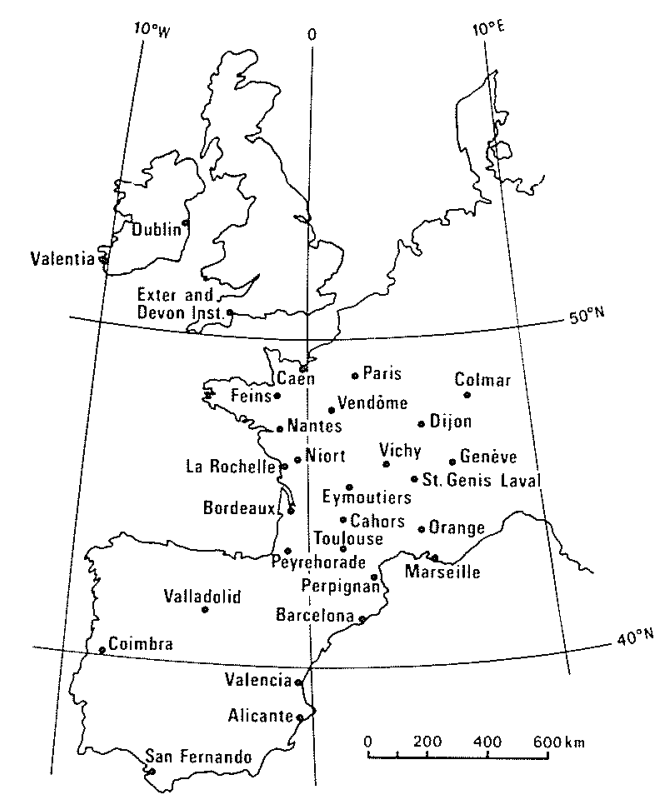

Figure 1 - Emplacement des stations pluviométriques étudiées. pour la France, au recueil de longues séries de mesures de précipitations (M. Garnier, 1974), pour l'Angleterre à la publication anglaise, Annual rainfall in England since 1725 (Craddock, 1976), puis complétée par les publications du Meteorological Office irlandais, de l'Instituto geofisico de Coimbra au Portugal et du Service Météorologique National de l'Espagne.

Cette recherche paraît d'autant plus intéressante que cet espace étudié présente une situation remarquable par rapport à la circulation atmosphérique générale des latitudes moyennes. La façade occidentale du continent européen reçoit directement les perturbations accompagnant les grands flux d'Ouest. Celles-ci apportent l'essentiel des précipitations dans le domaine océanique. La mise en évidence de périodes à forte pluviosité et de périodes à faible pluviosité sera à relier dans les études ultérieures aux fluctuations de l'intensité de cette circulation zonale d'Ouest.

\section{Réseau de mesures, méthodes d'analyse}

Considérée avec son ordre de succession dans le temps, la série des précipitations mensuelles, saisonnières ou annuelles à une station donnée constitue une série chronologique. Un grand nombre de techniques statistiques ont été développées pour tester le caractère aléatoire ou organisé d'une telle série et pour analyser la nature de cette éventuelle organisation. A titre d'exemple, nous citerons les travaux de Kiveliovitch et Vialar $(1953,1954,1955,1957)$ et le rapport technique de R. Sneyers (1975) exposant de façon détaillée les tests du caractère aléatoire simple des séries d'observations. Appliqués aux séries pluviométriques de l'Europe, en général ces tests ne font 
pas ressortir d'organisation très marquée (Kiveliovitch et Vialar, 1954), (Sneyers, 1979). Par exemple, si l'on considère les précipitations annuelles de Feins (Bretagne) sur la période 1860-1975, le test des maximums et des phases conduit à rejeter l'hypothèse d'une organisation temporelle de cette série (intervalle de confiance à 0.95 ).

Ce travail se situe dans une phase préliminaire à savoir une meilleure caractérisation des fluctuations pluviométriques observées par la prise en compte de leurs extensions spatiales et de leurs aspects saisonniers et non dans l'optique de tests systématiques visant à conclure au caractère aléatoire ou "organisé" de chacune des séries. En outre, après avoir pris un maximum de précautions quant à l'homogénéité des mesures à partir de la documentation disponible sur l'historique des stations, nous avons préféré dégager des résultats de l'observation conjointe d'un grand nombre de stations plutôt que de l'analyse fine d'une ou quelques stations réputées sûres. Pour cela, deux types d'analyses seront utilisés : le cumul des écarts normés et l'analyse factorielle.

\section{Méthode du cumul des écarts normés}

Le cumul des écarts à la moyenne à partir d'une date donnée fournit un moyen simple de caractériser l'évolution des séries climatologiques. Appliquée à la pluviométrie, cette méthode permet de décrire la succession des excès et des déficits et dans certains cas, de mieux comprendre l'influence des précipitations sur certains phénomènes naturels évoluant de façon cumulative (taille d'un arbre, épaisseur d'une langue glaciaire...).

Soit $p_{i}$ la série des précipitations mensuelles, saisonnières ou annuelles au cours de $n$ années consécutives $(i=1, n), m$ la moyenne calculée sur une période donnée commune à toutes les stations (de l'année $k$ à l'année $l$ ), et $c_{j}$ le cumul des écarts normés:

$$
m=\frac{1}{l-k+1} \sum_{i=k}^{l} p_{i} ; c_{j}=\frac{1}{m} \sum_{i=1}^{j}\left(p_{i}-m\right)
$$

Portés chronologiquement sur un graphique, les $c_{j}$ constituent la courbe des écarts cumulés normés (voir Fig. 2). Ce type d'analyse simple du comportement d'une série chronologique no sera pas utilisé à titre de test mais la courbe ainsi obtenue permettra de repérer graphiquement les périodes déficitaires ou excédentaires et de chiffrer rapidement en pourcentage de la moyenne ce déficit ou cet excédent. Contrairement à la technique des moyennes mobiles, chaque année conserve sa caractéristique pluviométrique puisque $p_{j}$ est proportionnel à $\left(c_{j}-c_{j-1}\right)$; par contre le graphique obtenu est très sensible à la valeur de la moyenne de référence et une hétérogénéité dans la série affectant cette moyenne peut modifier de façon importante le résultat final.

Pour pallier cet inconvénient nous avons fixé une période commune à toutes les stations pour le calcul de la moyenne de référence (1880-1960) et n'ont été retenues que les stations ne comportant pas à notre connaissance de déplacement majeur du site de la mesure.

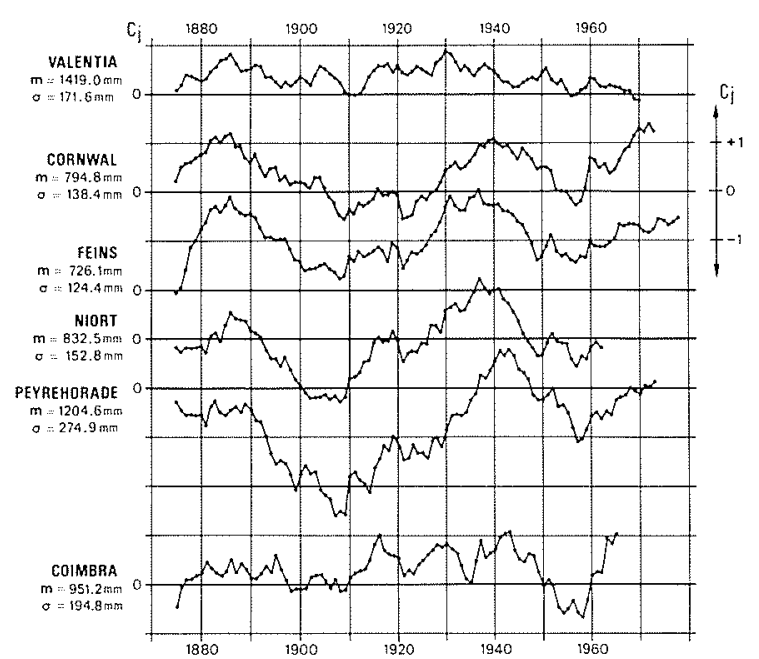

Figure 2 - Cumul des ćcarts normés des précipitations annuelles $\left(p_{i}\right)$

$$
c_{j}=\frac{1}{m} \sum_{i=1}^{j}\left(p_{i}-m\right)
$$

$m:$ moyenne des $p_{i}$

$\sigma$ : écart-type des $p_{i}$

de 1880 à 1960

\section{Méthode d'analyse factorielle en composantes prin- cipales $(A C P)$}

Les séries pluviométriques enregistrées dans des sites voisins sont corrélées entre elles; l'analyse en composantes principales permet alors de caractériser globalement l'évolution du réseau en travaillant non plus sur les stations séparément, mais sur de nouvelles variables qui sont des combinaisons linéaires des variables initiales. Pour un nombre d'observations donné ( $n$ années) et au cours d'un mois, d'une saison ou d'une année, les précipitations recueillies dans $m$ stations peuvent se représenter sous la forme d'une matrice $m \times n$. C'est l'information contenue dans cette matrice que nous étudierons par l'ACP.

Chaque variable standardisée $z_{j i}$ peut s'exprimer comme une combinaison linéaire de $p$ facteur $F_{k}$ communs à toutes les variables et d'un résidu $\epsilon_{i j}$.

$$
z_{j i}=\frac{p_{j i}-m_{j}}{\sigma_{j}}=a_{j 1} F_{1 i}+a_{j 2} F_{2 i}+\ldots a_{j p} F_{p i}+\epsilon_{i j}
$$

$p_{j i}=$ précipitation caractérisant l'année $i$ à la station $j$ $m_{j}, \sigma_{j}=$ moyenne, écart-type des précipitations à la station $j$.

Les $p$ facteurs sont orthogonaux entre eux ; ils s'expriment comme des combinaisons linéaires des variables de départ de façon à ce que la corrélation entre ces variables soit respectée aussi bien que possible. Les coefficients $a_{j k}$ sont linéairement proportionnels aux coefficients de corrélation $r_{i k}$ (corrélation entre la série des $z_{j i}$ et la série des $F_{k i}$ ).

Une analyse chronologique des $F_{k i}$ permettra donc une description synthétique des fluctuations pluviométriques sur l'ensemble du réseau ; les coefficients $r_{j k}$ mettent en évidence la cohérence ou alors les disparités dans la réaction des différentes stations. 


\section{Variations des précipitations annuelles}

\section{Les variations de l'ensemble des stations océaniques}

Les cumuls d'écarts normés pour un échantillon de stations choisies en Irlande, dans le Sud-Ouest de l'Angleterre et sur les façades atlantiques de la France et du Portugal, permettent de décrire l'évolution de la pluviométrie annuelle (Fig. 2). Dans les commentaires qui suivent, les déficits et les excédents pluviométriques sont donc exprimés en pourcentage de la moyenne calculée au cours de la période 1880-1960. De la Cornouaille (Angleterre) au Sud-Ouest de la France on note un déficit pluviométrique moyen de l'ordre de $10 \%$ au cours de la période 1887-1909; un excédent moyen de l'ordre de $8 \%$ sur la période 1922-1941 ; un déficit moyen de $10 \%$ de 1942 à 1957 et une légère reprise depuis 1958. L'examen des autres stations (Vendôme, Nantes, La Rochelle, Bordeaux) non représentées sur la figure faute de place, confirme ces résultats. Seule la station de Caen plus au Nord présente une différence au cours de la période 1942 1957 avec un écart cumulé nul à la place du déficit habituel.
Les précipitations de Valentia (Irlande) ne présentent pas de fluctuations importantes ni de synchronisme avec celles des autres stations. L'examen de la série de Dublin semble confirmer un comportement différencié des stations irlandaises.

De même, les précipitations de Coimbra (Portugal) ne présentent pas d'analogie avec l'ensemble des autres stations sauf sur la période récente (1933-1965). Ne disposant pas d'une autre longue série séculaire sur la façade littorale de la Péninsule Ibérique, il nous est difficile de conclure à une évolution climatique différente ou à une hétérogénéité de la série.

\section{Comparaisons avec les stations des régions climatiques océaniques dégradées ou méditerranéeennes}

Une étude similaire menée sur les stations de Colmar, Paris-St-Maur, Toulouse et Valladolid fait apparaître les mêmes phases pluviométriques déficitaires et excédentaires décrites dans le paragraphe précédent ; ceci implique une extension des résultats précédents sur un domaine géographique plus vaste que les seules zones littorales océaniques. Par contre, le Sud-Est (St GenisLaval par exemple), et surtout le littoral méditerranéen,

\begin{tabular}{|c|c|c|c|c|c|c|c|c|c|c|}
\hline & \multicolumn{2}{|c|}{ Année } & \multicolumn{2}{|c|}{ mars-mai } & \multicolumn{2}{|c|}{ juin-août } & \multicolumn{2}{|c|}{ sept-novemb. } & \multicolumn{2}{|c|}{ déc.-février } \\
\hline & $F_{1}$ & $F_{2}$ & $F_{1}$ & $F_{2}$ & $F_{1}$ & $F_{2}$ & $F_{1}$ & $\mathrm{~F}_{2}$ & $\mathrm{~F}_{1}$ & $F_{2}$ \\
\hline $\begin{array}{c}\text { Pourcentage } \\
\text { d'inertie }\end{array}$ & 38.3 & 9.9 & 37.2 & 11.7 & 35.9 & 10.3 & 38.9 & 12.5 & 51.5 & 10.5 \\
\hline Dublin & 0.51 & 0.05 & 0.49 & -0.14 & 0.57 & -0.40 & 0.57 & 0.07 & 0.61 & 0.11 \\
\hline Valentia & 0.25 & -0.09 & 0.46 & -0.17 & 0.26 & -0.52 & 0.36 & 0.20 & 0.50 & 0.00 \\
\hline Caen & 0.71 & -0.24 & 0.72 & -0.14 & 0.74 & -0.36 & 0.73 & -0.30 & 0.80 & -0.26 \\
\hline Feins & 0.80 & -0.30 & 0.82 & -0.30 & 0.76 & -0.32 & 0.80 & -0.17 & 0.87 & -0.25 \\
\hline Nantes & 0.78 & -0.22 & 0.80 & -0.21 & 0.74 & -0.31 & 0.83 & -0.12 & 0.86 & -0.17 \\
\hline Niort & 0.83 & -0.15 & 0.79 & -0.22 & 0.71 & -0.05 & 0.88 & -0.06 & 0.94 & -0.10 \\
\hline Peyrehorade & 0.74 & 0.02 & 0.72 & 0.14 & 0.71 & 0.20 & 0.62 & -0.04 & 0.74 & -0.14 \\
\hline Paris-St Maur & 0.77 & -0.12 & 0.78 & -0.18 & 0.73 & -0.08 & 0.76 & -0.30 & 0.85 & -0.14 \\
\hline Vendôme & 0.84 & -0.16 & 0.84 & -0.27 & 0.77 & -0.21 & 0.85 & -0.14 & 0.88 & -0.19 \\
\hline Eymoutiers & 0.80 & -0.02 & 0.80 & -0.12 & 0.75 & 0.07 & 0.89 & -0.02 & 0.86 & -0.06 \\
\hline Cahors & 0.68 & 0.07 & 0.70 & 0.19 & 0.65 & 0.31 & 0.74 & 0.19 & 0.87 & -0.03 \\
\hline Toulouse & 0.60 & 0.43 & 0.66 & 0.37 & 0.48 & 0.66 & 0.50 & 0.41 & 0.78 & -0.03 \\
\hline Coimbra & 0.52 & 0.05 & 0.51 & 0.16 & 0.39 & 0.25 & 0.34 & 0.39 & 0.79 & 0.33 \\
\hline San Fernando & 0.08 & 0.08 & 0.04 & 0.23 & 0.07 & 0.07 & 0.05 & 0.49 & 0.24 & 0.72 \\
\hline Valladolid & 0.42 & 0.00 & 0.30 & 0.13 & 0.01 & 0.28 & 0.30 & 0.46 & 0.74 & 0.26 \\
\hline Colmar & 0.67 & 0.05 & 0.48 & 0.00 & 0.71 & 0.03 & 0.73 & -0.24 & 0.76 & 0.02 \\
\hline Dijon & 0.80 & 0.14 & 0.75 & -0.09 & 0.79 & 0.10 & 0.82 & -0.16 & 0.88 & -0.01 \\
\hline Genève & 0.80 & 0.01 & 0.74 & -0.15 & 0.72 & -0.11 & 0.79 & -0.16 & 0.90 & 0.07 \\
\hline Vichy & 0.73 & 0.14 & 0.70 & 0.04 & 0.75 & 0.03 & 0.74 & 0.10 & 0.66 & -0.12 \\
\hline St Genis Laval & 0.72 & 0.22 & 0.71 & 0.15 & 0.80 & -0.06 & 0.63 & 0.27 & 0.79 & 0.29 \\
\hline Orange & 0.29 & 0.32 & 0.38 & 0.58 & 0.49 & 0.09 & 0.39 & 0.52 & 0.46 & 0.58 \\
\hline Marseille & 0.32 & 0.72 & 0.27 & 0.70 & 0.40 & 0.61 & 0.31 & 0.68 & 0.51 & 0.58 \\
\hline Perpignan & -0.05 & 0.75 & 0.23 & 0.67 & 0.32 & 0.62 & 0.04 & 0.67 & -0.21 & 0.53 \\
\hline Barcelone & 0.00 & 0.78 & 0.20 & 0.78 & 0.23 & 0.51 & 0.02 & 0.73 & -0.10 & 0.68 \\
\hline Alicante & -0.31 & 0.33 & 0.00 & 0.60 & 0.03 & 0.06 & -0.38 & 0.32 & -0.35 & 0.40 \\
\hline
\end{tabular}


(Barcelone par exemple), sont caractérisés par des fluctuations différentes. Pour obtenir un résultat synthétique à partir d'un plus grand nombre de stations il est alors nécessaire de recourir aux méthodes d'analyse multivariée.

Une analyse en composantes principales des précipitations annuelles de 25 stations pluviométriques (Tabl. I) sur la plus grande période commune de relevés (18811962) fait apparaître un premier facteur $F_{1}$ bien corrélé avec des séries pluviométriques de stations océaniques de l'Ouest de la France et des stations du Nord-Est. La corrélation est plus faible avec les stations de l'Irlande et de l'Ouest de l'Espagne et du Portugal, voire négative pour les stations méditerranéennes et du Sud de l'Espagne. Compte tenu du plus grand nombre de stations de type océanique ou soumises à l'action des perturbations d'Ouest, à ce facteur correspond un fort pourcentage d'explication de la variance totale $(38 \%)$. Le second facteur est bien corrélé avec les séries des stations méditerranéennes (Marseille, Perpignan, Barcelone) ; son pouvoir explicatif est assez faible (10\%).

$\mathrm{La}$ représentation des fluctuations au cours des années des coefficients $F_{1 i}$ peut se faire par leur cumul chronologique. A des valeurs $F_{1 i}$ positives correspondent des précipitations excédentaires dans les stations océaniques et aux valeurs négatives de $F_{1 i}$ des précipitations déficitaires. Le graphique obtenu (Fig. 3, année) confirme et généralise les résultats de la comparaison des courbes d'écarts cumulés normés tracées pour chacune des stations.

Par ailleurs, une application de l'analyse factorielle des correspondances (J.P. Benzecri, 1980) dont l'exposé ne peut être développé dans les limites de cet article suggère pour l'ensemble des régions océaniques

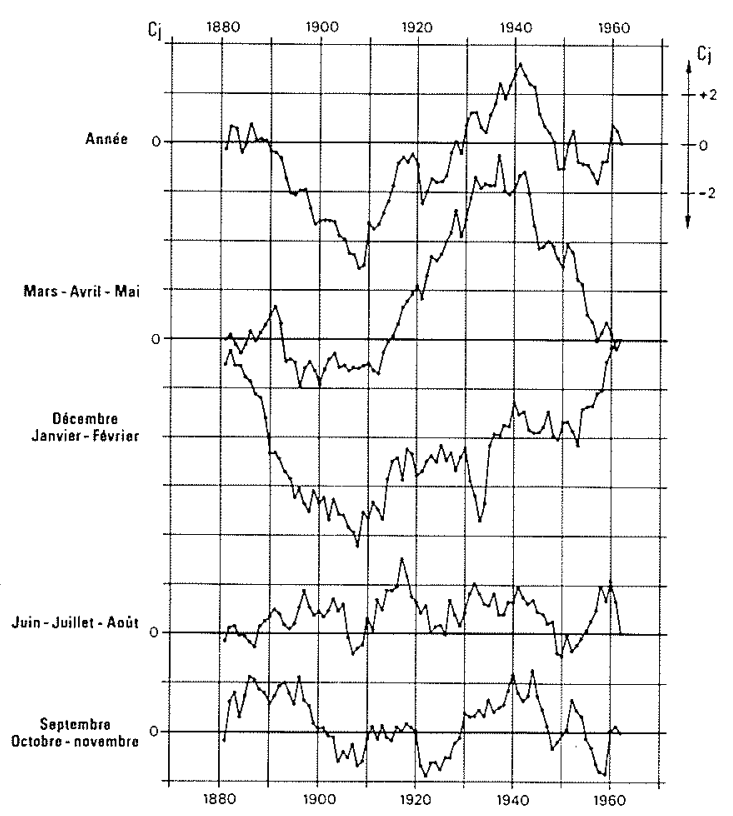

Figure 3 - Cumuls chronologiques des facteurs $F_{1 i}$ calculés à partir d'ACP des précipitations annuelles puis saisonnières de 25 stations sur la période 1881-1962

$$
c_{j}=\sum_{i=1}^{j} F_{1 i}
$$

une organisation spatiale des précipitations en années contrastées (années très pluvieuses 1886, 1910, 1927... années sèches 1870, 1888, 1921, 1949).

Ainsi, à l'échelle annuelle et pour la majeure partie de la façade atlantique (Irlande exceptée), la comparaison de l'évolution chronologique des séries pluviométriques de chaque station et les résultats des analyses factorielles effectuées mettent donc en évidence un synchronisme dans leurs comportements. La question qui se pose maintenant est de savoir si ces variations annuelles sont le résultat d'une superposition de variations saisonnières différentes ou bien si elles sont influencées par des fluctuations communes de mois ou de groupes de mois.

\section{Le ròle des variations saisonnières}

\section{Description des variations saisonnières}

Les résultats des analyses en composantes principales saisonnières et le cumul chronologique des facteurs ainsi obtenus (Tab. I, saisons et Fig. 3) amènent les remarques suivantes:

- le printemps est caractérisé par deux périodes très différenciées : 1910-1942 avec un excédent moyen qui atteint $20 \%$ à Peyrehorade et 1943-1957 avec un déficit moyen de l'ordre de $20 \%$. Les moyennes pluviométriques caractérisant deux périodes consécutives d'au moins 15 ans peuvent donc présenter des écarts de l'ordre de $40 \%$;

- l'été et l'automne ne présentent pas d'écarts importants entre périodes d'années consécutives ;

- l'hiver se caractérise par un déficit moyen supérieur à $15 \%$ de 1875 à 1919 , soit un écart de plus de $25 \%$ entre la précipitation moyenne de cette période et celle de la période 1911-1970.

En outre les analyses en composantes principales portant sur les précipitations de printemps, d'été et d'automne (Tab. I), font apparaître la même régionalisation que dans le cas annuel avec des pourcentages d'explication de la variance très voisins. Les précipitations de l'hiver se différencient avec une plus forte cohérence spatiale (fortes corrélations entre les séries des stations influencées par les perturbations océaniques) ce qui conduit à un fort pourcentage d'explication de la variance de $F_{1}(52 \%)$. A l'exception des stations méditerranéennes et du Sud de l'Espagne, toutes sont fortement corrélées avec ce facteur. Coimbra, Valladolid et Dublin présentent alors les mêmes fluctuations que celles enregistrées dans les autres stations océaniques (déficit jusqu'en 1909).

\section{L 'influence de l'hiver et du printemps}

Compte tenu de leurs fortes variations au cours du siècle écoulé, les précipitations recueillies au cours de ces deux saisons influenceront de façon importante les fluctuations du total annuel. Ainsi, le déficit moyen enregistré de 1875 à 1909 s'explique essentiellement par. la faiblesse des précipitations hivernales ; celui de la période 1942-1957 par la faiblesse des précipitations 


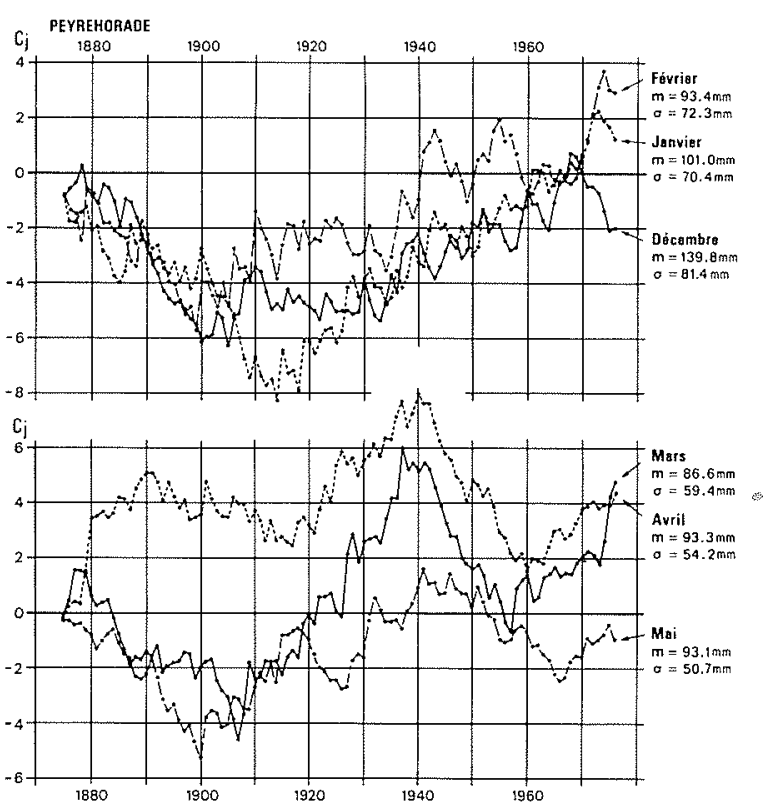

Figure 4 - Cumuls des écarts normés des précipitations mensuelles recueillies à Peyrehorade.

$$
c_{j}=\frac{1}{m} \sum_{i=1}^{j}\left(p_{i}-m\right)
$$

$m:$ moyenne des $p_{i}$ $\sigma:$ écart-type des $p_{i}$

$$
\text { de } 1880-1960
$$

printanières ; l'excédent de la période 1922-1941 résulte de fortes précipitations printanières.

Si l'on veut affiner l'étude au niveau du mois, le travail devient fastidieux. La variabilité interannuelle des précipitations devient très forte et l'interprétation des résultats délicate. Néanmoins, pour l'hiver et le printemps, les courbes du cumul des écarts normés tracés pour chacun des mois font ressortir les mêmes fluctuations d'ensemble que la saison à laquelle ces mois appartiennent. Ce fait est bien illustré par la station de Peyrehorade (Fig. 4) ; on aboutit aux mêmes conclusions par l'étude mensuelle de la plupart des autres stations océaniques. Ce résultat nous paraît important car il semble indiquer qu'au moins pour ces deux saisons et sur la période d'étude, les fluctuations d'ensemble ne sont pas la superposition de trois évolutions mensuelles différentes mais que la même tendance au déficit ou à un excédent au cours d'une période donnée a touché l'ensemble de la saison.

\section{Conclusion}

L'étude chronologique de longues séries pluviométriques réparties sur la façade atlantique de l'Europe a permis de dégager les traits communs à leurs variations au cours des cent dernières années. Au niveau des précipitations annuelles de l'hiver et du printemps, la plus grande partie de cette façade (Irlande et Sud de l'Espagne exceptées), présente des fluctuations communes. Les principales phases pluviométriques différenciées sont les suivantes :

- affaiblissement des précipitations annuelles de 1887 à 1909 (déficit $>15 \%$ pour l'hiver) ;

- période plus arrosée en moyenne de 1922 à 1941 (excédents de l'ordre de $8 \%$ pour l'année et de $20 \%$ pour le printemps) ;

- période plus sèche que la normale de 1942 à 1957 (déficits moyens de l'ordre de $10 \%$ pour l'année et de $20 \%$ pour le printemps).

L'analyse conjointe d'un grand nombre de stations situées dans différents pays semble exclure les seules hypothèses d'hétérogénéités dues à des changements du site de la mesure, de l'appareillage ou du mode opératoire. Les résultats obtenus devront être confrontés avec les fluctuations de la fréquence des grands types de la circulation atmosphérique à l'échelle synoptique.

\section{Bibliographie, sources}

BENZECRI J.P. - Pratique de l'analyse des données. Dunod Paris $1980,424 \mathrm{p}$.

CRADDOCK J.M. - Annual rainfall in England since 1725. Quaterly Journal of the Royal Meteorolcgical Society, 1976,102 , p. 823-840.

GARNIER M. - Longues séries de mesures de précipitations en France, Memorial de la Météorologie Nationale. Paris 1974, Fasc. 1, 109 p., Fasc. 2, 122 p., Fasc. 3, 114 p., Fasc. 4, 130 p.

INSTITUTO GEOFISICO DE COIMBRA. - Normais e valores dos elementos principais. Instituto Geofisico de Coimbra, 2 fasc., $1942,1955$.

KIVELIOVITCH M., VIALAR J. - Comment déceler la présence du hasard dans l'observation des phénomènes naturels. La Météorologie, avril-juin 1954, p. 151-170.

KIVELIOVITCH M., VIALAR j. - Etude statistique des séries chronologiques (J. Scient. Météorologie, t. V, n ${ }^{\circ} 17,18$, $19,20,1953$; t. VI, n $21,23,24,1954$; t. VII, n 27 , 1955 ; t. IX, n $33,36,1957$ ) ; séries chronologiques et le hasard (Publ. scient. techn. Ministère de l'Air, ${ }^{\circ}$ NT 65, 1957).

MARCHAND J.P. - La variabilité de l'organisation mensuelle des précipitations. L'exemple de Dublin-Phoenix Park (18381976). "Eaux et Climats", Mélanges Géographiques offerts en hommage à Ch. P. Peguy. Publication E.R. 30 du C.N.R.S. Université Scient. et Méd. de Grenoble, 1981.

MOUNIER J. - Les climats océaniques des régions atlantiques de l'Espagne et du Portugal. Thèse d'Etat Lille III, 1979. 2 tomes, 1221 p. +1 Atlas 246 p. et index, 14 cartes h. texte.

PEGUY Ch. P. - Ordre et désordre des climats. L'Espace Géographique, $1979 / 1$, p. 5-14

SERVICE METEOROLOGIQUE NATIONAL ESPAGNOL. Las series mas largas de observaciones pluviometricas en la Peninsula Iberica. Pub. Serie D. n $1,1943$.

SNEYERS R. - Sur l'analyse statistique des séries d'observations. Note technique $\mathrm{n}^{\circ} 143$, Secrétariat de l'Organisation Météorologique Mondiale. Genève, 1975, $193 \mathrm{p}$.

SNEYERS R. - Homogénéité et stabilité des éléments météorologiques à Uccle (Belgique), Il Nuovo Cimento, 1979, Vol. 2, $\mathrm{n}^{\circ} 1$

VIALAR J. - Calcul des probabilités et statistique. Tome IV Statistique et étude des séries chronologiques, 1978. Direction de la Météorologie Nationale, Paris, $128 \mathrm{p}$ 


\section{Discussion}

Président : M. M. ROCHE

Le Président. - Je remercie M. MOUNIER et M. MARTIN de leur très intéressant exposé et je vais demander si quelqu'un a des remarques, des commentaires à faire, ou des questions à poser.

M. GROSSE. --. L'homogénéité des séries publiées par la Météorologie est douteuse. Les sites et appareils de mesure ont souvent changé. L'historique accompagnant ces publications est insuffisant. Il serait intéressant, comme l'ont fait des équipes anglaises, d'essayer de produire, par des études régionales ou locales des séries "homogènes", corrigées, autant que possible, des effets des changements de matériel et de site, en s'appuyant sur toute l'information disponible.

M. MOUNIER. - Il est certain que pour des stations étudiées, ici, il nous a manqué certaines précisions concernant les sites, les types d'appareil ... Cependant, nous avons fait un choix parmi les séries séculaires que nous avons archivées, à partir de la présentation des données et des renseignements sur les localisations. Ainsi, pour la France de l'Ouest, nous avons retenu Feins et Nantes seulement. D'autre part, si la station de Coîmbra, de l'Institut de Géophysique offre des données fiables, l'utilisation des longues séries publiées par la Météorologie espagnole est plus délicate, par suite des changements fréquents des localisations de nombreuses stations.

M. MARTIN. - Nous sommes bien conscients que, malgré les précautions qui ont été prises, il peut subsister des causes d'hétérogénéité dans les séries retenues (évolution de l'environnement du site de la mesure, changement d'appareillage, changement du mode opératoire, etc.).

C'est pourquoi nous avons fait porter nos conclusions sur l'étude comparative d'un grand nombre de stations et non pas sur les résultats issus de l'analyse d'une, deux ou trois stations seulement. En outre, la similitude des résultats issus de l'analyse des séries françaises et de ceux obtenus à partir de certaines des séries anglaises "homogénéisées" telle la série caractéristique du Devon et de la Cornouaille (Craddock J.M., 1976) semble confirmer nos conclusions.

Le Président. - Je voudrais ajouter quelque chose. Je crois que ce que M. GROSSE a voulu dire n'a pas été tout à fait perç. En fait, je pense que son propos n'était pas simplement de faire les tests pour éliminer des stations considérées comme déficientes, mais de rechercher quelles étaient les corrections à apporter pour qu'on ait à chaque station, des séries qui soient homogènes dans le temps.

M. THIRRIOT. - Très intéressé par votre exposé et ne connaissant rien à la méthode des écarts cumulés, j'ai essayé de me faire une idée en considérant un écart non aléatoire mais systématique suivant une loi sinusoïdale.

$\mathrm{Si}$

$\mathrm{Si}$

$$
\begin{gathered}
\epsilon=a \sin \omega t \\
\Sigma \epsilon \rightarrow \frac{a}{\omega}(1-\cos \omega t) \\
\epsilon=a \sin (\omega t+\varphi) \\
\Sigma \epsilon \rightarrow \frac{a}{\omega}(\cos \varphi-\cos (\omega t+\varphi))
\end{gathered}
$$

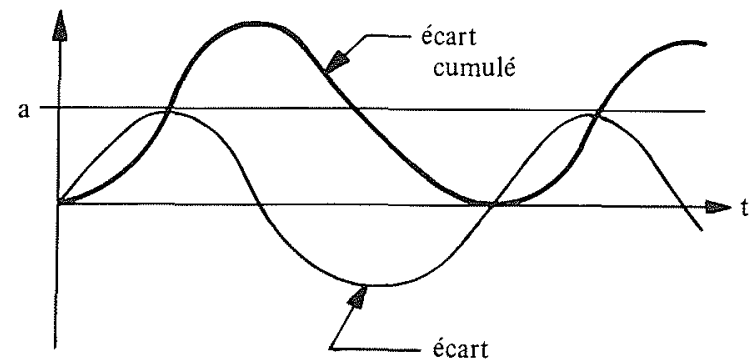

Donc la position du graphe écart cumulé par rapport à l'axe des temps peut être située tout au dessus (si $\varphi=0$ ), tout au-dessous $(\varphi=\pi)$ ou de part et d'autre de l'axe.

Partant de l'analogie déterministe ci-dessus mes questions seront les suivantes:

1) quand le graphe des écarts cumulés, est tout du même côté peut-on déduire de son amplitude $\frac{2 a}{\omega}$ l'amplitude moyenne des écarts non cumulés (ceci reste vrai d'ailleurs s'il y a une phase initiale donc d'une manière générale que dire de l'utilisation possible de l'amplitude apparente $\frac{a}{\omega}=\frac{a T}{2 \pi}$ ),

2) y a t-il une signification physique à la différence de phase $\varphi$ entre stations (en postulant même période de la série des écarts cumulés). Peut-on retenir la remarque de quadrature de phase pour les séries aléatoires?

Enfin si on considère une somme de sinusoïdes pour l'écart

$$
=a_{1} \sin \left(\omega_{1 t}+\varphi_{1}\right)+a_{2} \sin \left(\omega_{2} t+\varphi_{2}\right)
$$

I'écart cumulé est

$$
\begin{aligned}
\Sigma \epsilon \rightarrow \frac{a_{1}}{\omega_{1}}\left(\cos \varphi_{1}-\cos \left(\omega_{1} t\right.\right. & \left.+\varphi_{1}\right)+ \\
& +\frac{a_{2}}{\omega_{2}}\left[\cos \varphi_{2}-\cos \left(\omega_{2} t+\varphi_{2}\right)\right]
\end{aligned}
$$

Si $\omega_{2} \gg \omega_{1}$ le second terme qui est analogue à un bruit est étouffé

C'est l'effet de lissage dû à l'intégration (à la différence de l'opération de dérivation). Peut-on multiplier les sommations (prendre la cumulée de l'écart cumulé, etc.)?

M. MARTIN. - Je répondrai en disant que dans le cadre de ce travail, nous avons utilisé la méthode de cumul des écarts normés dans une optique essentiellement descriptive et non pas comme une méthode d'analyse du signal recherchant et caractérisant des variations pluviométriques de type sinusoïdal; nous avons voulu mettre en évidence de façon graphique et relativement simple des périodes au cours desquelles la pluviométrie est excédentaire et des périodes au cours desquelles la pluviométrie est déficitaire. L'allure générale de la courbe dépendra de la position dans le temps, de la durée et de l'intensité de ces périodes (au sens nombre d'années consécutives) excédentaires ou déficitaires.

Le Président. - La moyenne prise pour faire le calcul de l'écart cumulé n'est pas la moyenne prise sur la période d'observation de la station, mais une période standard adoptée pour toutes les stations. C'est sans doute ce qui donne une allure un peu bizarre à la courbe.

M. DER MEGREDITCHIAN. - Je voudrais dire quelques mots au sujet de la réalité physique de cet effet.

D'abord, en ce qui concerne l'observation suivant laquelle il y a des stations qui sont soit tout en-dessous, soit tout au-dessus, c'est une chose qui est très bien étudiée et qui est tout simplement due à ce qu'on appelle l'effet des lois d'Arsinus. Même si l'on considère qu'on a un phénomène aléatoire avec probabilité de $1 / 2$ en-dessous ou au-dessus, donc déficitaire ou excédentaire, si l'on considère ce qu'on appelle la période de temps passé au-dessus ou au-dessous, c'est dû à la loi d'Arsinus, c'est-à-dire une loi en $U$. C'est-à-dire que les courbes les moins fréquentes sont celles pour lesquelles on passe autant de temps au-dessus qu'en-dessous. Par conséquent les courbes les plus fréquentes d'intensité étant nulles, c'est soit tout en-dessous, soit tout au-dessus, ce qui est contradictoire à ce qu'on appelle l'effet gaussien. Dans ce cas, les courbes normales sont les moins fréquentes, à la différence du cas gaussien, où les courbes normales sont les plus fréquentes. 
Je voudrais poser une question : vous n'avez pas pensé à étudier ce qu'on peut appeler la longueur d'onde, le nombre de fois où l'on coupe l'axe, la longueur moyenne, etc. en utilisant précisément l'appareil des lois d'Arsinus, la loi sur le temps passé au-dessous ou au-dessus et la loi sur la longueur moyenne? Dans cet esprit, il serait intéressant, pour chaque station, de connaître la longueur moyenne d'une onde de la partie au-dessus, la période excédentaire ou la période déficitaire. C'est une caractérisation importante, et on peut utiliser dans ce domaine l'appareil des lois d'Arsinus.

M. MARTIN. - Je pense que les méthodes statistiques appropriées doivent permettre de pousser plus loin l'analyse chronologique des séries pluviométriques mais deux types de problèmes vont se poser : collecte de séries pluri-séculaires si l'on veut aboutir à des résultats significatifs et choix du paramètre à analyser. Va-t-on faire des tests systématiques sur chacune des stations; sur la moyenne de l'ensemble des stations étudiées, sur les facteurs issus d'analyses en composantes principales ou sur les fréquences de types de situations météorologiques responsables de précipitations?

A quelle échelle faut-il travailler ? Le mois, la saison, l'année ? Je crois que le problème se pose à ce niveau et c'est pourquoi un travail préliminaire relativement descriptif et synthétique est un préalable nécessaire pour essayer de définir dans quel sens et sur quelles variables il faut pousser l'analyse plus avant.

M. GUILLOT. - L'examen des lignes d'écarts-cumulés est un moyen suffisamment robuste et significatif pour ne pas rendre indispensables des procédés mathématiquement plus érudits, mais qui apportent sans doute peu en plus (on pourrait laisser MM. THIRRIOT et DER MEGREDITCHIAN discuter entre-eux là-dessus, et lire Hurst, Feller, Mandelbrot, etc.) Pour l'hydrologue, la question intéressante est de savoir s'il s'est agi seulement d'une dérive des procédés de mesure, ou d'un comportement réel du climat de l'Ouest Europe. Les procédés de mesure étant indépendants dans les 3 pays Espagne, France, Royaume Uni, on est donc bien conduit à admettre qu'il s'agit d'un phénomène climatique réel, que personne ne sait expliquer à ce jour (mais qui est bien conforme aux constatations de Hurst). Je serais beaucoup plus circonspect sur la cassure 1950 des stations savoyardes comme le Thyl et Valmenier, où l'importance relative de la neige dans le total des précipitations laisse craindre un effet des modifications de l'appareillage et des modes opératoires, après l'intervention d'EDF sur le réseau climato (cuve à neige, table à neige, puis seau profond).

M. BEDIOT. - Nous savions, avec M. GOUBET, qu'en inscrivant cette question à l'ordre du jour du Comité Technique celà allait susciter des débats passionnés.

Je voudrais apporter un certain nombre d'éléments qui datent d'il y a quinze ou seize ans. J'avais obtenu les manuscrits des sériers de Garnier, dont il a été question tout à l'heure, 35 séries qui concernaient le bassin Seine-Normandie. A la demande de M. TIXERONT, j'avais fait des moyennes mobiles de trente ans. On pouvait constater, pour la presque totalité, que la moyenne de 1870 à 1899 (je cite de mémoire) était la plus faible et que la moyenne de 1910 à 1939 était la plus forte. Il y avait, pour certains postes, une amplitude de $100 \mathrm{~mm}$.

Cela pose un certain nombre de problèmes, d'une part, pourquoi $\mathrm{y}$-a-t-il une certaine concordance entre les 35 stations ? D'autre part, lorsqu'on réalise des aménagements où il s'agit simplement de dimensionner des barrages-réservoirs pour accumuler de l'eau, en assurant un remplissage complet, 9 années sur 10 et que les bassins versants d'alimentation se situent dans une tranche de pluviométrie où tout $\mathrm{mm}$ supplémentaire ruisselle en quasi-totalité, la question de la représentativité de la période d'observation utilisée se pose vis à vis des fluctuations enregistrées au cours des 150 dernières années. En effet, un écart de $100 \mathrm{~mm}$ entraine dans ces conditions un écart de $3 \mathrm{l} / \mathrm{s} / \mathrm{km}^{2}$ sur le module spécifique ce qui est énorme pour un bassin de plaine.

C'était déjà une remarque que je m'étais faite à l'époque puis je l'ai laissée dans un coin de ma mémoire et, de temps à autre - comme aujourd'hui - elle ressort.

Ce qui m'a beaucoup intéressé dans ce qui a été présenté par MM. MOUNIER et MARTIN, c'est leur décomposition mois par mois, qu'a soulignée un peu M. GUILLOT. Pour la période antérieure à 1900 , on constate une baisse des précipitations en hiver et, par contre, pour la période suivante, on constate une élévation des précipitations en mai et juin. Je suis d'accord avec M. GUILLOT, il faut faire très attention; il y aurait une petite enquête à faire sur le nombre de jours de neige et les températures de ces hivers-là; il y a eu un certain nombre d'hivers remarquablement froids durant la période de 1870 à 1900 , et il y a peut-être une explication du côté des précipitations neigeuses.

S'il y a des précipitations excédentaires sur mai et juin, cela ne se remarque pas d'une manière extraordinaire sur les écoulements annuels, puisque, en gros, c'est repris par l'évaporation. A l'occasion d'une étude assez récente faite avec $M$. MASSON cette remarque a resurgi, puisqu'on a constaté que la série des débits antérieurs à 1900 , reconstituée à partir des hauteurs d'eau relevées à l'échelle limnimétrique de Bar-surSeine fournissait un module inférieur à celui de la série postérieure à 1900 .

Il y a là toute une série de faits qui montrent l'intérêt des recherches historiques sur les données du passé. Je pense que M. GUILLOT est tout à fait d'accord avec moi pour que l'on recherche aussi tout ce qui concerne les événements exceptionnels observés jadis. Mais il faut parallèlement essayer de tirer au clair ces variations amples, constatées au cours des 150 dernières années.

Le Président. - Je remercie M. BEDIOT. Je vois que cette communication a suscité beaucoup de commentaires.

Nous sommes tenus maintenant de passer à la communication suivante. 


\section{Abstract \\ Changes in rain fall conditiones in western Europe over the last century}

This paper presents an analysis of the rainfall fluctuations during the last century over the West part of Europe (Fig. 1). Our purpose is not to apply systematic random tests but to caracterize the principal changes common to large areas of annual and seasonal rainfall series.

The main sources of information on monthly and annual rainfall to tals are :

- Meteorologie Nationale (Garnier, 1974) in France

- Institu to Geofisico de Coimbra (1942-1955) in Portugal

- Service meteorologique national (1943) in Spain

Annual rainfall in England since 1725 (Craddock, 1976).

Two techniques have been applied to caracterize rainfall variations : for each individual station the mass curve (accumulated departures from the average of consecutive rainfall totals expressed as percentage of the average) and principal components analysis for the whole network.

Ireland and South Spain excepted, the oceanic coastal regions of Europe show common changes in their annual rainfall records (Fig. 2. Fig. 3) : a 10\% mean deficiency during the period $1887-1909$ and $1042-1957$; a $8 \%$ mean excess from 1922 to 1941 and a low excess since 1958. If some other stations of the inland (Paris, Colmar, Toulouse,...) exhibit the same evolution, on the other hand mediterrean stations show completely different variations. Further investigations thanks to seasonal analysis (Fig. 3) show that the deficiency of 1887-1909 is mainly due to a winter deficiency; the excess of 1922-1941 and the successive deficiency (1942-1957) result from important contrast in their mean spring rainfalls ( $40 \%$ of difference in some stations). For winter and spring the two seasons which are caracterized by noticeable changes in their pluviometric evolution, each monthly rainfall serie indicate the same similar evolution as that of the seasonal total (Fig. 4).

These results are related to changes in the general circulation of the atmosphere during the last century and espacially to variations in the strength of the zonal circulation. 